\title{
Existence of Positive Solutions to a Nonlocal Boundary Value Problem with $p$-Laplacian on Time Scales
}

\author{
Ting-Ting Sun, Lin-Lin Wang, and Yong-Hong Fan
}

School of Mathematics and Information, Ludong University, Yantai, Shandong 264025, China

Correspondence should be addressed to Lin-Lin Wang, wangll_1994@sina.com

Received 6 October 2009; Accepted 19 January 2010

Academic Editor: Paul Eloe

Copyright (C) 2010 Ting-Ting Sun et al. This is an open access article distributed under the Creative Commons Attribution License, which permits unrestricted use, distribution, and reproduction in any medium, provided the original work is properly cited.

The nonlocal boundary value problem, with $p$-Laplacian of the form $\left(\Phi_{p}\left(u^{\Delta}\right)\right)^{\Delta}(t)+h(t) f(t, u(t))=$ $0, t \in\left[t_{1}, t_{m}\right)_{\mathbb{T}}, u^{\Delta}\left(t_{1}\right)-\sum_{j=1}^{n} \theta_{j} u^{\Delta}\left(\eta_{j}\right)-\sum_{i=1}^{m-2} \epsilon_{i} u\left(\xi_{i}\right)=0, u^{\Delta}\left(t_{m}\right)=0$, has been considered. Two existence criteria of at least one and three positive solutions are presented. The first one is based on the Four functionals fixed point theorem in the work of R. Avery et al. (2008), and the second one is based on the Five functionals fixed point theorem. Meanwhile an example is worked out to illustrate the main result.

\section{Introduction}

Due to the unification of the theory of differential and difference equations, there have been many investigations working on the existence of positive solutions to boundary value problems for dynamic equations on time scales. Also there is much attention paid to the study of multipoint boundary value problem with $p$-Laplacian; see [1-10].

For convenience, throughout this paper we denote $\Phi_{p}(s)$ as the $p$-Laplacian operator, that is, $\Phi_{p}(s)=|s|^{p-2} s, p>1$. $\left(\Phi_{p}\right)^{-1}=\Phi_{q}$, where $1 / p+1 / q=1$.

In [11], the author discussed the positive solutions of a $m$-point boundary value problem for a second-order dynamic equation on a time scale

$$
\begin{gathered}
u^{\Delta \nabla}(t)+q(t) f(u(t))=0, \quad t \in[0, T]_{\mathbb{T}} \\
u^{\Delta}(0)=\sum_{i=1}^{m-2} b_{i} u^{\Delta}\left(\xi_{i}\right), \quad u(T)=\sum_{i=1}^{m-2} a_{i} u\left(\xi_{i}\right),
\end{gathered}
$$


where $a_{i}, b_{i} \geq 0(i=1,2, \ldots, m-2)$, and $\xi_{i} \in(0, \rho(T))_{\mathbb{T}}$ with $0<\xi_{1}<\xi_{2}<\cdots<\xi_{m-2}<\rho(T)$. And he got the existence of at least two positive solutions of the above problem by means of a fixed point theorem in a cone.

Zhao and Ge [9] considered the following multi-point boundary value problem with one-dimensional $p$-Laplacian:

$$
\begin{gathered}
\left(\Phi_{p}\left(x^{\prime}(t)\right)\right)^{\prime}+f(t, x(t))=0, \quad 0<t<1, \\
x^{\prime}(0)-\sum_{i=1}^{m-1} a_{i} x\left(\xi_{i}\right)=0, \quad x^{\prime}(1)+\sum_{i=1}^{m-1} \beta_{i} x\left(\eta_{i}\right)=0,
\end{gathered}
$$

where $a_{i}, \beta_{i}>0,0<\sum_{i=1}^{m-1} a_{i} \xi_{i} \leq 1,0<\sum_{i=1}^{m-1} \beta_{i}\left(1-\eta_{i}\right) \leq 1, i=1,2, \ldots, m-1,0=\xi_{1}<\xi_{2}<\cdots<$ $\xi_{m-1}<\eta_{1}<\eta_{2}<\cdots<\eta_{m-1}=1$. By using a fixed point theorem in a cone, they obtained the existence of at least one, two, or three positive solutions under some sufficient conditions.

Motivated by the above results, in this paper, we investigate the nonlocal boundary value problem with $p$-Laplacian

$$
\begin{gathered}
\left(\Phi_{p}\left(u^{\Delta}\right)\right)^{\Delta}(t)+h(t) f(t, u(t))=0, \quad t \in\left[t_{1}, t_{m}\right)_{\mathbb{T}} \\
u^{\Delta}\left(t_{1}\right)-\sum_{j=1}^{n} \theta_{j} u^{\Delta}\left(\eta_{j}\right)-\sum_{i=1}^{m-2} \epsilon_{i} u\left(\xi_{i}\right)=0, \quad u^{\Delta}\left(t_{m}\right)=0,
\end{gathered}
$$

where $0 \leq t_{1}<\xi_{1}<\xi_{2}<\cdots<\xi_{m-2}<t_{m}$ and $t_{1}<\eta_{1}<\eta_{2}<\cdots<\eta_{n}<t_{m}<+\infty$.

For convenience, we list the following hypotheses:

(H1) $\epsilon_{i}>0, i=1,2, \ldots, m-2, \theta_{j} \geq 0, j=1,2, \ldots, n, \sum_{i=1}^{m-2} \epsilon_{i} \xi_{i}+\sum_{j=1}^{n} \theta_{j}<1$;

(H2) $f(t, u) \in C\left(\left[t_{1}, t_{m}\right]_{\mathbb{T}} \times[0,+\infty),[0,+\infty)\right)$ and $f$ is not identically zero on any compact subinterval of $\left[t_{1}, t_{m}\right]_{\mathbb{T}} \times[0,+\infty)$;

(H3) $h(t) \in C_{r d}\left(\left[t_{1}, t_{m}\right]_{\mathbb{T}},[0,+\infty)\right)$ and $h$ is not identically zero on any compact subinterval of $\left[t_{1}, t_{m}\right]_{\mathbb{T}}$, also it satisfies

$$
\Phi_{q}\left(\int_{t_{1}}^{t_{m}} h(\tau) \Delta \tau\right)<+\infty, \quad \int_{t_{1}}^{\sigma\left(t_{m}\right)} \Phi_{q}\left(\int_{s}^{t_{m}} h(\tau) \Delta \tau\right) \Delta s<+\infty .
$$

By using the Four functionals fixed point theorem and Five functionals fixed point theorem, we obtain the existence criteria of at least one positive solution and three positive solutions for the BVP (1.3). As an application, an example is worked out finally. The remainder of this paper is organized as follows. Section 2 is devoted to some preliminary discussions. We give and prove our main results in Section 3.

\section{Preliminaries}

The basic definitions and notations on time scales can be found in [12,13]. In the following, we will provide some background materials on the theory of cones in Banach spaces. For more details, please refer to $[14,15]$. 
Definition 2.1. Let $E$ be a Banach space. A nonempty, closed set $P \subset E$ is said to be a cone provided that the following hypotheses are satisfied:

(1) if $x, y \in P, \alpha, \beta \geq 0$, then $\alpha x+\beta y \in P$;

(2) if $x \in P, x \neq \theta$, then $x \in P$. $y-x \in P$.

Every cone $P \subset E$ induces a partial ordering " $\leq$ " on $E$ defined by $x \leq y$ if and only if

Definition 2.2. A map $\alpha$ is said to be a nonnegative continuous concave functional on a cone $P$ of a real Banach space $E$ if $\alpha: P \rightarrow[0, \infty)$ is continuous and $\alpha(t x+(1-t) y) \geq t \alpha(x)+(1-t) \alpha(y)$ for all $x, y \in P$ and $t \in[0,1]$. Similarly, we say that the map $\beta$ is a nonnegative continuous convex functional on a cone $P$ of a real Banach space $E$ if $\beta: P \rightarrow[0, \infty)$ is continuous and $\beta(t x+(1-t) y) \leq t \beta(x)+(1-t) \beta(y)$ for all $x, y \in P$ and $t \in[0,1]$.

Let $\alpha$ and $\Psi$ be nonnegative continuous concave functionals on $P$, and let $\beta$ and $\theta$ be nonnegative continuous convex functionals on $P$; then for positive numbers $r, l, v$, and $R$, define the sets

$$
\begin{gathered}
Q(\alpha, \beta, r, R)=\{x \in P: r \leq \alpha(x), \beta(x) \leq R\}, \\
U(\Psi, \iota)=\{x \in Q(\alpha, \beta, r, R): \iota \leq \Psi(x)\}, \\
V(\theta, v)=\{x \in Q(\alpha, \beta, r, R): \theta(x) \leq v\} .
\end{gathered}
$$

The following lemma can be found in [16].

Lemma 2.3 (four functionals fixed point theorem). If $P$ is a cone in a real Banach space $E, \alpha$ and $\Psi$ are nonnegative continuous concave functionals on $P, \beta$, and $\theta$ are nonnegative continuous convex functionals on $P$, and there exist nonnegative positive numbers $r, l, v$, and $R$, such that

$$
A: Q(\alpha, \beta, r, R) \longrightarrow P
$$

is a completely continuous operator, and $Q(\alpha, \beta, r, R)$ is a bounded set. If

(i) $\{x \in U(\Psi, \iota): \beta(x)<R\} \cap\{x \in V(\theta, v): r<\alpha(x)\} \neq \emptyset$,

(ii) $\alpha(A x) \geq r$, for all $x \in Q(\alpha, \beta, r, R)$, with $\alpha(x)=r$ and $v<\theta(A x)$,

(iii) $\alpha(A x) \geq r$, for all $x \in V(\theta, v)$, with $\alpha(x)=r$,

(iv) $\beta(A x) \leq R$, for all $x \in Q(\alpha, \beta, r, R)$, with $\beta(x)=R$ and $\Psi(A x)<\iota$,

(v) $\beta(A x) \leq R$, for all $x \in U(\Psi, \iota)$, with $\beta(x)=R$,

then $A$ has a fixed point $x$ in $Q(\alpha, \beta, r, R)$. 
We are now in a position to present the Five functionals fixed point theorem (see [17]). Let $\gamma, \beta, \theta$ be nonnegative continuous convex functionals on $P$ and $\alpha, \varphi$ nonnegative continuous concave functionals on $P$. For nonnegative numbers $h, a, b, d$, and $c$, define the following convex sets:

$$
\begin{gathered}
P(\gamma, c)=\{x \in P: \gamma(x)<c\}, \\
P(\gamma, \alpha, a, c)=\{x \in P: a \leq \alpha(x), \gamma(x) \leq c\}, \\
Q(\gamma, \beta, d, c)=\{x \in P: \beta(x) \leq d, \gamma(x) \leq c\}, \\
P(\gamma, \theta, \alpha, a, b, c)=\{x \in P: a \leq \alpha(x), \theta(x) \leq b, \gamma(x) \leq c\}, \\
Q(\gamma, \beta, \varphi, h, d, c)=\{x \in P: h \leq \varphi(x), \beta(x) \leq d, \gamma(x) \leq c\} .
\end{gathered}
$$

Lemma 2.4 (five functionals fixed point theorem). Let $P$ be a cone in a real Banach space $E$. Suppose that there exist nonnegative numbers $c$ and $M$, nonnegative continuous concave functionals $\alpha$ and $\varphi$ on $P$, and nonnegative continuous convex functionals $\gamma, \beta$, and $\theta$ on $P$, with

$$
\alpha(x) \leq \beta(x), \quad\|x\| \leq M \gamma(x) \quad \forall x \in \overline{P(\gamma, c)} .
$$

Suppose that $A: \overline{P(\gamma, c)} \rightarrow \overline{P(\gamma, c)}$ is completely continuous and there exist nonnegative numbers $h, a, k, b$, with $0<a<b$ such that

(i) $\{x \in P(\gamma, \theta, \alpha, b, k, c): \alpha(x)>b\} \neq \emptyset$ and $\alpha(A(x))>b$ for $x \in P(\gamma, \theta, \alpha, b, k, c)$,

(ii) $\{x \in Q(\gamma, \beta, \varphi, h, a, c): \beta(x)<a\} \neq \emptyset$ and $\beta(A(x))<a$ for $x \in Q(\gamma, \beta, \varphi, h, a, c)$,

(iii) $\alpha(A(x))>b$ for $x \in P(\gamma, \alpha, b, c)$ with $\theta(A(x))>k$,

(iv) $\beta(A(x))<$ a for $x \in Q(\gamma, \beta, a, c)$ with $\varphi(A(x))<h$,

then $A$ has at least three fixed points $x_{1}, x_{2}, x_{3} \in \overline{P(\gamma, c)}$ such that

$$
\beta\left(x_{1}\right)<a, \quad \alpha\left(x_{2}\right)>b, \quad \beta\left(x_{3}\right)>a \quad \text { with } \alpha\left(x_{3}\right)<b .
$$

Consider the Banach space $E=C\left[t_{1}, \sigma\left(t_{m}\right)\right]_{\mathbb{T}}$ equipped with the norm $\|u\|=$ $\max _{t \in\left[t_{1}, \sigma\left(t_{m}\right)\right]_{\mathbb{T}}}|u(t)|$. Suppose $\rho, \eta \in \mathbb{T}$ with $t_{1}<\rho<\eta<\sigma\left(t_{m}\right)$. For the sake of convenience, we take the notations

$$
\begin{gathered}
\varpi=\sum_{i=1}^{m-2} \epsilon_{i}, \quad h_{0}=\Phi_{q}\left(\int_{t_{1}}^{t_{m}} h(\tau) \Delta \tau\right), \quad M_{0}=\int_{t_{1}}^{Q} \Phi_{q}\left(\int_{\rho}^{\eta} h(\tau) \Delta \tau\right) \Delta s, \\
M_{Q}=\int_{t_{1}}^{\rho} \Phi_{q}\left(\int_{s}^{t_{m}} h(\tau) \Delta \tau\right) \Delta s, \\
M_{\eta}=\int_{t_{1}}^{\eta} \Phi_{q}\left(\int_{s}^{t_{m}} h(\tau) \Delta \tau\right) \triangle s, \quad M_{\sigma\left(t_{m}\right)}=\int_{t_{1}}^{\sigma\left(t_{m}\right)} \Phi_{q}\left(\int_{s}^{t_{m}} h(\tau) \Delta \tau\right) \triangle s .
\end{gathered}
$$


Define a cone

$$
\begin{aligned}
& P=\left\{u \in E: u(t) \geq 0, u^{\Delta}\left(t_{1}\right)-\sum_{j=1}^{n} \theta_{j} u^{\Delta}\left(\eta_{j}\right)-\sum_{i=1}^{m-2} \epsilon_{i} u\left(\xi_{i}\right)=0,\right. \\
& \left.\quad \text { for } t \in\left[t_{1}, \sigma\left(t_{m}\right)\right]_{\mathbb{T}} \text { and } u^{\Delta \Delta}(t) \leq 0, u^{\Delta}(t) \geq 0 \text { for } t \in\left[t_{1}, t_{m}\right)_{\mathbb{T}}, u^{\Delta}\left(t_{m}\right)=0\right\}
\end{aligned}
$$

and an operator $A: P \rightarrow E$ by

$$
\begin{aligned}
A u= & \frac{\Phi_{q}\left(\int_{t_{1}}^{t_{m}} h(\tau) f(\tau, u(\tau)) \Delta \tau\right)}{\varpi}-\frac{\sum_{i=1}^{m-2} \epsilon_{i} \int_{t_{1}}^{\xi_{i}} \Phi_{q}\left(\int_{s}^{t_{m}} h(\tau) f(\tau, u(\tau)) \Delta \tau\right) \Delta s}{\varpi} \\
& -\frac{\sum_{j=1}^{n} \theta_{j} \Phi_{q}\left(\int_{\eta_{j}}^{t_{m}} h(\tau) f(\tau, u(\tau)) \Delta \tau\right)}{\varpi}+\int_{t_{1}}^{t} \Phi_{q}\left(\int_{s}^{t_{m}} h(\tau) f(\tau, u(\tau)) \Delta \tau\right) \Delta s .
\end{aligned}
$$

Lemma 2.5. $A: P \rightarrow P$.

Proof. For $u \in P, t \in\left[t_{1}, \sigma\left(t_{m}\right)\right]_{\mathbb{T}}$,

$$
\begin{aligned}
A u(t) \geq & \frac{1-\sum_{j=1}^{n} \theta_{j}-\sum_{i=1}^{m-2} \epsilon_{i} \xi_{i}}{\varpi} \Phi_{q}\left(\int_{t_{1}}^{t_{m}} h(\tau) f(\tau, u(\tau)) \Delta \tau\right) \\
& +\int_{t_{1}}^{t} \Phi_{q}\left(\int_{s}^{t_{m}} h(\tau) f(\tau, u(\tau)) \Delta \tau\right) \Delta s \\
\geq & 0 .
\end{aligned}
$$

From the definition of $A$, it is clear that

$$
(A u)^{\Delta}(t)=\Phi_{q}\left(\int_{t}^{t_{m}} h(s) f(s, u(s)) \triangle s\right) \geq 0, \quad t \in\left[t_{1}, t_{m}\right]_{\mathbb{T}},
$$

is continuous, $(A u)^{\Delta}\left(t_{1}\right)-\sum_{j=1}^{n} \theta_{j}(A u)^{\Delta}\left(\eta_{j}\right)-\sum_{i=1}^{m-2} \epsilon_{i}(A u)\left(\xi_{i}\right)=0$, and $(A u)\left(\sigma\left(t_{m}\right)\right)$ is the maximum value of $A u(t)$ on $\left[t_{1}, \sigma\left(t_{m}\right)\right]_{\mathbb{T}}$.

Let $g(t)=\int_{t}^{t_{m}} h(s) f(s, u(s)) \triangle s$, then $g: \mathbb{R} \rightarrow \mathbb{R}$ is continuous, $g: \mathbb{T} \rightarrow \mathbb{R}$ is delta differentiable on $\left[t_{1}, t_{m}\right]_{\mathbb{T}^{k}}$, and $\Phi_{q}: \mathbb{R} \rightarrow \mathbb{R}$ is continuously differentiable. Moreover $\Phi_{q}(s)$ is monotonically increasing for $s \geq 0$ and $g^{\Delta}(t)=-h(t) f(t, u(t)) \leq 0$. Then by the chain rule [12, Theorem 1.87, page 31], we obtain

$$
(A u)^{\Delta \Delta}(t)=\Phi_{q}^{\prime}(g(c)) g^{\triangle}(t) \leq 0,
$$

where $c$ is in the interval $[t, \sigma(t)]$. So, $A: P \rightarrow P$. This completes the proof. 


\section{Main Results and an Example}

Theorem 3.1. Assume that (H1), (H2), and (H3) hold, if there exist constants $r, l, v, R$ with $R>$ $\max \left\{\left(h_{0}+\varpi M_{\sigma\left(t_{m}\right)}\right) / \varpi M_{0},\left(\sigma\left(t_{m}\right)-t_{1}\right) /\left(\rho-t_{1}\right)\right\} \iota, v \geq \max \left\{\left(\left(h_{0}+\varpi M_{\eta}\right) / \varpi M_{\rho}\right) \iota,\left(\left(\eta-t_{1}\right) /(\rho-\right.\right.$ $\left.\left.\left.t_{1}\right)\right) r\right\}, r<\iota$ and suppose that $f(t, u)$ satisfies the following conditions:

(A1) $f(t, u) \leq \Phi_{p}\left(R \varpi /\left(h_{0}+\varpi M_{\sigma\left(t_{m}\right)}\right)\right)$ for all $(t, u) \in\left[t_{1}, t_{m}\right]_{\mathbb{T}} \times[0, R]$,

(A2) $f(t, u) \geq \Phi_{p}\left(r / M_{0}\right)$ for all $(t, u) \in[\rho, \eta]_{\mathbb{T}} \times[r, v]$,

then the BVP (1.3) has a fixed point $u \in P$ such that

$$
\min _{t \in[\rho, \eta]_{\mathbb{T}}} u(t) \geq r, \quad \max _{t \in\left[t_{1}, \sigma\left(t_{m}\right)\right]_{\mathbb{T}}} u(t) \leq R
$$

Define maps

$$
\alpha(u)=\Psi(u)=\min _{t \in[\rho, \eta]_{\mathbb{T}}} u(t), \quad \theta(u)=\max _{t \in[\rho, \eta]_{\mathbb{T}}} u(t), \quad \beta(u)=\max _{t \in\left[t_{1}, \sigma\left(t_{m}\right)\right]_{\mathbb{T}}} u(t),
$$

and let $Q(\alpha, \beta, r, R), U(\Psi, \iota)$ and $V(\theta, v)$ be defined by $(2.1)$. lemma.

In order to complete the proof of Theorem 3.1, we first need to prove the following

Lemma 3.2. $Q(\alpha, \beta, r, R)$ is bounded and $A: Q(\alpha, \beta, r, R) \rightarrow P$ is completely continuous.

Proof. For all $u \in Q(\alpha, \beta, r, R),\|u\|=\max _{t \in\left[t_{1}, \sigma\left(t_{m}\right)\right]_{\mathbb{T}}}|u(t)|=\beta(u) \leq R$, which means that $Q(\alpha, \beta, r, R)$ is a bounded set.

According to Lemma 2.5, it is clear that $A: Q(\alpha, \beta, r, R) \rightarrow P$.

In view of the continuity of $f$, there exists a constant $C>0$ such that $f(t, u)<\Phi_{p}(C)$, for all $t \in\left[t_{1}, \sigma\left(t_{m}\right)\right]_{\mathbb{T}}, u \in Q(\alpha, \beta, r, R)$. Consider

$$
\|A u\|=A u\left(\sigma\left(t_{m}\right)\right) \leq\left(\frac{h_{0}}{\varpi}+M_{\sigma\left(t_{m}\right)}\right) C,
$$

which means that $A Q(\alpha, \beta, r, R)$ is uniformly bounded.

In addition, for all $t_{1} \leq \bar{t} \leq t^{*} \leq \sigma\left(t_{m}\right)$, we have

$$
\left|A u(\bar{t})-A u\left(t^{*}\right)\right|=\left|\int_{\bar{t}}^{t^{*}} \Phi_{q}\left(\int_{s}^{t_{m}} h(\tau) f(\tau, u(\tau)) \Delta \tau\right) \Delta S\right| \leq C h_{0}\left|\bar{t}-t^{*}\right| .
$$

Applying the Arzelà-Ascoli theorem on time scales [18], one can show that $A Q(\alpha, \beta, r, R)$ is relatively compact.

Now we prove that $A: Q(\alpha, \beta, r, R) \rightarrow P$ is continuous. Let $\left\{u_{n}\right\}_{n \in \mathbb{N}}$ be a sequence in $Q(\alpha, \beta, r, R)$ which converges to $u_{0} \in Q(\alpha, \beta, r, R)$ uniformly on $\left[t_{1}, \sigma\left(t_{m}\right)\right]_{\mathbb{T}}$. Because 
$A Q(\alpha, \beta, r, R)$ is relatively compact, the sequence $\left\{A u_{n}\right\}$ admits a subsequence $\left\{A u_{n_{m}}\right\}$ converging to $v(t)$ uniformly on $\left[t_{1}, \sigma\left(t_{m}\right)\right]_{\mathbb{T}}$. In addition,

$$
0 \leq A u_{n}(t) \leq C\left(\frac{h_{0}}{\omega}+M_{\sigma\left(t_{m}\right)}\right)
$$

Observe that

$$
\begin{aligned}
A u_{n}(t)= & \frac{\Phi_{q}\left(\int_{t_{1}}^{t_{m}} h(\tau) f\left(\tau, u_{n}(\tau)\right) \Delta \tau\right)}{\varpi}-\frac{\sum_{i=1}^{m-2} \epsilon_{i} \int_{t_{1}}^{\xi_{i}} \Phi_{q}\left(\int_{s}^{t_{m}} h(\tau) f\left(\tau, u_{n}(\tau)\right) \Delta \tau\right) \Delta s}{\varpi} \\
& -\frac{\sum_{j=1}^{n} \theta_{j} \Phi_{q}\left(\int_{\eta_{j}}^{t_{m}} h(\tau) f\left(\tau, u_{n}(\tau)\right) \Delta \tau\right)}{\varpi}+\int_{t_{1}}^{t} \Phi_{q}\left(\int_{s}^{t_{m}} h(\tau) f\left(\tau, u_{n}(\tau)\right) \Delta \tau\right) \Delta s .
\end{aligned}
$$

Hence, by the Lebesgue's dominated convergence theorem on time scales [19], insert $u_{n_{m}}$ into the above equality and then let $m \rightarrow \infty$, we obtain

$$
\begin{aligned}
v(t)= & \frac{\Phi_{q}\left(\int_{t_{1}}^{t_{m}} h(\tau) f\left(\tau, u_{0}(\tau)\right) \Delta \tau\right)}{\varpi}-\frac{\sum_{i=1}^{m-2} \epsilon_{i} \int_{t_{1}}^{\xi_{i}} \Phi_{q}\left(\int_{s}^{t_{m}} h(\tau) f\left(\tau, u_{0}(\tau)\right) \Delta \tau\right) \Delta s}{\varpi} \\
& -\frac{\sum_{j=1}^{n} \theta_{j} \Phi_{q}\left(\int_{\eta_{j}}^{t_{m}} h(\tau) f\left(\tau, u_{0}(\tau)\right) \Delta \tau\right)}{\varpi}+\int_{t_{1}}^{t} \Phi_{q}\left(\int_{s}^{t_{m}} h(\tau) f\left(\tau, u_{0}(\tau)\right) \Delta \tau\right) \Delta s .
\end{aligned}
$$

From the definition of $A$, we know that $v(t)=A u_{0}(t)$ on $\left[t_{1}, \sigma\left(t_{m}\right)\right]_{\mathbb{T}}$. This shows that each subsequence of $\left\{A u_{n}(t)\right\}_{n=1}^{\infty}$ uniformly converges to $A u_{0}(t)$. Therefore the sequence $\left\{A u_{n}(t)\right\}_{n=1}^{\infty}$ uniformly converges to $A u_{0}(t)$. This means that $A$ is continuous at $u_{0} \in$ $Q(\alpha, \beta, r, R)$. So, $A$ is continuous on $Q(\alpha, \beta, r, R)$ since $u_{0}$ is arbitrary. Thus, $A: Q(\alpha, \beta, r, R) \rightarrow$ $P$ is completely continuous. This completes the proof.

Proof of Theorem 3.1. Let

$$
\begin{aligned}
u_{0}= & \frac{\iota}{M_{Q}}\left(\frac{\Phi_{q}\left(\int_{t_{1}}^{t_{m}} h(\tau) \Delta \tau\right)-\sum_{i=1}^{m-2} \epsilon_{i} \int_{t_{1}}^{\xi_{i}} \Phi_{q}\left(\int_{s}^{t_{m}} h(\tau) \Delta \tau\right) \Delta s}{\varpi}\right) \\
& -\frac{\iota}{M_{Q}}\left(\frac{\sum_{j=1}^{n} \theta_{j} \Phi_{q}\left(\int_{\eta_{j}}^{t_{m}} h(\tau) \Delta \tau\right)}{\varpi}\right)+\frac{\iota}{M_{\varrho}} \int_{t_{1}}^{t} \Phi_{q}\left(\int_{s}^{t_{m}} h(\tau) \Delta \tau\right) \Delta s .
\end{aligned}
$$


8

Advances in Difference Equations

Clearly, $u_{0} \in P$. By direct calculation,

$$
\begin{aligned}
& \Psi\left(u_{0}\right)=\frac{\iota}{M_{Q}}\left(\frac{\Phi_{q}\left(\int_{t_{1}}^{t_{m}} h(\tau) \Delta \tau\right)-\sum_{i=1}^{m-2} \epsilon_{i} \int_{t_{1}}^{\xi_{i}} \Phi_{q}\left(\int_{s}^{t_{m}} h(\tau) \Delta \tau\right) \Delta s}{\varpi}\right) \\
& -\frac{\iota}{M_{Q}}\left(\frac{\sum_{j=1}^{n} \theta_{j} \Phi_{q}\left(\int_{\eta_{j}}^{t_{m}} h(\tau) \Delta \tau\right)}{\varpi}\right)+\frac{\iota}{M_{Q}} \int_{t_{1}}^{\rho} \Phi_{q}\left(\int_{s}^{t_{m}} h(\tau) \Delta \tau\right) \triangle S \\
& \geq \frac{\iota}{M_{\rho}} \int_{t_{1}}^{\rho} \Phi_{q}\left(\int_{s}^{t_{m}} h(\tau) \Delta \tau\right) \Delta s=\iota, \\
& \beta\left(u_{0}\right)=\frac{\iota}{M_{Q}}\left(\frac{\Phi_{q}\left(\int_{t_{1}}^{t_{m}} h(\tau) \Delta \tau\right)-\sum_{i=1}^{m-2} \epsilon_{i} \int_{t_{1}}^{\xi_{i}} \Phi_{q}\left(\int_{s}^{t_{m}} h(\tau) \Delta \tau\right) \Delta s}{\varpi}\right) \\
& -\frac{\iota}{M_{Q}}\left(\frac{\sum_{j=1}^{n} \theta_{j} \Phi_{q}\left(\int_{\eta_{j}}^{t_{m}} h(\tau) \Delta \tau\right)}{\varpi}\right)+\frac{\iota}{M_{Q}} \int_{t_{1}}^{\sigma\left(t_{m}\right)} \Phi_{q}\left(\int_{s}^{t_{m}} h(\tau) \Delta \tau\right) \Delta s \\
& <\frac{\iota}{M_{Q}}\left(\frac{\Phi_{q}\left(\int_{t_{1}}^{t_{m}} h(\tau) \Delta \tau\right)}{\varpi}+\int_{t_{1}}^{\sigma\left(t_{m}\right)} \Phi_{q}\left(\int_{s}^{t_{m}} h(\tau) \Delta \tau\right) \Delta s\right) \leq R, \\
& \theta\left(u_{0}\right)=\frac{\iota}{M_{\rho}}\left(\frac{\Phi_{q}\left(\int_{t_{1}}^{t_{m}} h(\tau) \Delta \tau\right)-\sum_{i=1}^{m-2} \epsilon_{i} \int_{t_{1}}^{\xi_{i}} \Phi_{q}\left(\int_{s}^{t_{m}} h(\tau) \Delta \tau\right) \Delta s}{\varpi}\right) \\
& -\frac{\iota}{M_{Q}}\left(\frac{\sum_{j=1}^{n} \theta_{j} \Phi_{q}\left(\int_{\eta_{j}}^{t_{m}} h(\tau) \Delta \tau\right)}{\varpi}\right)+\frac{\iota}{M_{Q}} \int_{t_{1}}^{\eta} \Phi_{q}\left(\int_{s}^{t_{m}} h(\tau) \Delta \tau\right) \Delta s \\
& \leq \frac{\iota}{M_{Q}}\left(\frac{\Phi_{q}\left(\int_{t_{1}}^{t_{m}} h(\tau) \Delta \tau\right)}{\varpi}+\int_{t_{1}}^{\eta} \Phi_{q}\left(\int_{s}^{t_{m}} h(\tau) \Delta \tau\right) \Delta s\right) \leq v, \\
& \alpha\left(u_{0}\right)=\frac{\iota}{M_{Q}}\left(\frac{\Phi_{q}\left(\int_{t_{1}}^{t_{m}} h(\tau) \Delta \tau\right)-\sum_{i=1}^{m-2} \epsilon_{i} \int_{t_{1}}^{\xi_{i}} \Phi_{q}\left(\int_{s}^{t_{m}} h(\tau) \Delta \tau\right) \Delta s}{\varpi}\right) \\
& -\frac{\iota}{M_{Q}}\left(\frac{\sum_{j=1}^{n} \theta_{j} \Phi_{q}\left(\int_{\eta_{j}}^{t_{m}} h(\tau) \Delta \tau\right)}{\varpi}\right)+\frac{\iota}{M_{Q}} \int_{t_{1}}^{Q} \Phi_{q}\left(\int_{s}^{t_{m}} h(\tau) \Delta \tau\right) \Delta s \\
& >\frac{\iota}{M_{\rho}} \int_{t_{1}}^{\rho} \Phi_{q}\left(\int_{s}^{t_{m}} h(\tau) \Delta \tau\right) \Delta s>r .
\end{aligned}
$$


So, $u_{0} \in\{u \in U(\Psi, \iota): \beta(u)<R\} \cap\{u \in V(\theta, v): r<\alpha(u)\}$, which means that (i) in Lemma 2.3 is satisfied.

For all $u \in Q(\alpha, \beta, r, R)$, with $\alpha(u)=r$ and $v<\theta(A u)$, we have $\alpha(A u)=A u(\rho) \geq$ $\left(\left(\rho-t_{1}\right) /\left(\eta-t_{1}\right)\right) A u(\eta)=\left(\left(\rho-t_{1}\right) /\left(\eta-t_{1}\right)\right) \theta(A u)>\left(\left(\rho-t_{1}\right) /\left(\eta-t_{1}\right)\right) v>r$, and for all $u \in Q(\alpha, \beta, r, R)$, with $\beta(u)=R$ and $\Psi(A u)<\iota$, we obtain that $\beta(A u)=A u\left(\sigma\left(t_{m}\right)\right) \leq\left(\left(\sigma\left(t_{m}\right)-\right.\right.$ $\left.\left.t_{1}\right) /\left(\rho-t_{1}\right)\right) A u(\rho)=\left(\left(\sigma\left(t_{m}\right)-t_{1}\right) /\left(\rho-t_{1}\right)\right) \Psi(A u)<\left(\left(\sigma\left(t_{m}\right)-t_{1}\right) /\left(\rho-t_{1}\right)\right) \iota<R$. Hence, (ii) and (iv) in Lemma 2.3 are fulfilled.

For any $u \in V(\theta, v)$, with $\alpha(u)=r$,

$$
\begin{aligned}
\alpha(A u)= & \frac{\Phi_{q}\left(\int_{t_{1}}^{t_{m}} h(\tau) f(\tau, u(\tau)) \Delta \tau\right)-\sum_{i=1}^{m-2} \epsilon_{i} \int_{t_{1}}^{\xi_{i}} \Phi_{q}\left(\int_{s}^{t_{m}} h(\tau) f(\tau, u(\tau)) \Delta \tau\right) \Delta s}{\varpi} \\
& -\frac{\sum_{j=1}^{n-2} \theta_{j} \Phi_{q}\left(\int_{\eta_{j}}^{t_{m}} h(\tau) f(\tau, u(\tau)) \Delta \tau\right)}{\varpi}+\int_{t_{1}}^{\varrho} \Phi_{q}\left(\int_{s}^{t_{m}} h(\tau) f(\tau, u(\tau)) \Delta \tau\right) \Delta s \\
\geq & \int_{t_{1}}^{Q} \Phi_{q}\left(\int_{\rho}^{\eta} h(\tau) f(\tau, u(\tau)) \Delta \tau\right) \Delta s \geq \int_{t_{1}}^{\rho} \Phi_{q}\left(\int_{\rho}^{\eta} h(\tau) \Phi_{p}\left(\frac{r}{M_{0}}\right) \Delta \tau\right) \Delta s=r,
\end{aligned}
$$

and for all $u \in U(\Psi, \iota)$, with $\beta(u)=R$,

$$
\begin{aligned}
\beta(A u)= & \frac{\Phi_{q}\left(\int_{t_{1}}^{t_{m}} h(\tau) f(\tau, u(\tau)) \Delta \tau\right)-\sum_{i=1}^{m-2} \epsilon_{i} \int_{t_{1}}^{\xi_{i}} \Phi_{q}\left(\int_{s}^{t_{m}} h(\tau) f(\tau, u(\tau)) \Delta \tau\right) \Delta s}{\varpi} \\
& -\frac{\sum_{j=1}^{n-2} \theta_{j} \Phi_{q}\left(\int_{\eta_{j}}^{t_{m}} h(\tau) f(\tau, u(\tau)) \Delta \tau\right)}{\varpi}+\int_{t_{1}}^{\eta} \Phi_{q}\left(\int_{s}^{t_{m}} h(\tau) f(\tau, u(\tau)) \Delta \tau\right) \Delta s \\
\leq & \frac{\Phi_{q}\left(\int_{t_{1}}^{t_{m}} h(\tau) \Phi_{p}\left(R \varpi /\left(h_{0}+\varpi M_{\sigma\left(t_{m}\right)}\right) \Delta \tau\right)\right.}{\varpi} \\
& +\int_{t_{1}}^{\sigma\left(t_{m}\right)} \Phi_{q}\left(\int_{s}^{t_{m}} h(\tau) \Phi_{p}\left(\frac{R \varpi}{h_{0}+\varpi M_{\sigma\left(t_{m}\right)}}\right) \Delta \tau\right) \Delta s \\
= & R .
\end{aligned}
$$

Thus (iii) and (v) in Lemma 2.3 hold true. So, by Lemma 2.3, the BVP (1.3) has a fixed point $u$ in $Q(\alpha, \beta, r, R)$. This completes the proof.

Theorem 3.3. Assume that (H1), (H2), and (H3) hold. If there exist constants $h, a, b, c, k$, with $b<$ $a \varpi M_{0} /\left(h_{0}+\varpi M_{\eta}\right), c>\left(\left(h_{0}+\varpi M_{\sigma\left(t_{m}\right)}\right) /\left(h_{0}+\varpi M_{\eta}\right)\right) a, k<\left(\left(h_{0}+\varpi M_{\eta}\right) /\left(h_{0}+\varpi M_{\sigma\left(t_{m}\right)}\right)\right) c$, $k \geq \max \left\{\left(\eta-t_{1}\right) /\left(\rho-t_{1}\right),\left(h_{0}+\varpi M_{\eta}\right) / \varpi M_{\varphi}\right\} b, a \geq \max \left\{\left(\eta-t_{1}\right) /\left(\rho-t_{1}\right),\left(h_{0}+\varpi M_{\eta}\right) / \varpi M_{\varphi}\right\} h$, further suppose that $f(t, u)$ satisfies the following conditions:

(B1) $f(t, u)<\Phi_{p}\left(a \varpi /\left(h_{0}+\varpi M_{\eta}\right)\right)$ for all $(t, u) \in\left[t_{1}, t_{m}\right]_{\mathbb{T}} \times[0, c]$,

(B2) $f(t, u) \geq \Phi_{p}\left(b / M_{0}\right)$ for all $(t, u) \in[\rho, \eta]_{\mathbb{T}} \times[b, k]$, 
then the BVP (1.3) has at least three positive solutions $u_{1}, u_{2}$ and $u_{3}$ such that

$$
\max _{t \in[\rho, \eta]_{\mathbb{T}}} u_{1}(t)<a<\max _{t \in[\rho, \eta]_{\mathbb{T}}} u_{3}(t), \quad \min _{t \in[\rho, \eta]_{\mathbb{T}}} u_{3}(t)<b<\min _{t \in[\rho, \eta]_{\mathbb{T}}} u_{2}(t)
$$

Proof. Define these maps

$$
\alpha(u)=\varphi(u)=\min _{t \in[\rho, \eta]} u(t), \quad \beta(u)=\theta(u)=\max _{t \in[\rho, \eta]} u(t), \quad \gamma(u)=\max _{t \in\left[t_{1}, \sigma\left(t_{m}\right)\right]} u(t),
$$

and let $P(\gamma, c), P(\gamma, \alpha, b, c), Q(\gamma, \beta, a, c), P(\gamma, \theta, \alpha, b, k, c)$ and $Q(\gamma, \beta, \varphi, h, a, c)$ be defined by (2.3). It is clear that

$$
\alpha(u) \leq \beta(u), \quad\|u\| \leq \gamma(u), \quad \forall u \in \overline{P(\gamma, c)} .
$$

Using similar methods as those in Lemma 3.2, we obtain that $A: \overline{P(\gamma, c)} \rightarrow P$ is completely continuous. Thus, we only need to show that $A: \overline{P(\gamma, c)} \rightarrow \overline{P(\gamma, c)}$. Let $u \in$ $\overline{P(\gamma, c)}$, then

$$
\begin{aligned}
r(A u)= & \frac{\Phi_{q}\left(\int_{t_{1}}^{t_{m}} h(\tau) f(\tau, u(\tau)) \Delta \tau\right)}{\varpi}-\frac{\sum_{i=1}^{m-2} \epsilon_{i} \int_{t_{1}}^{\xi_{i}} \Phi_{q}\left(\int_{s}^{t_{m}} h(\tau) f(\tau, u(\tau)) \Delta \tau\right) \Delta s}{\varpi} \\
& -\frac{\sum_{j=1}^{n} \theta_{j} \Phi_{q}\left(\int_{\eta_{j}}^{t_{m}} h(\tau) f(\tau, u(\tau)) \Delta \tau\right)}{\varpi}+\int_{t_{1}}^{\sigma\left(t_{m}\right)} \Phi_{q}\left(\int_{s}^{t_{m}} h(\tau) f(\tau, u(\tau)) \Delta \tau\right) \Delta s \\
\leq & \frac{\Phi_{q}\left(\int_{t_{1}}^{t_{m}} h(\tau) f(\tau, u(\tau)) \Delta \tau\right)}{\varpi}+\int_{t_{1}}^{\sigma\left(t_{m}\right)} \Phi_{q}\left(\int_{s}^{t_{m}} h(\tau) f(\tau, u(\tau)) \Delta \tau\right) \Delta s \\
\leq & \frac{\Phi_{q}\left(\int_{t_{1}}^{t_{m}} h(\tau) \Phi_{p}\left(a \varpi /\left(h_{0}+\varpi M_{\eta}\right)\right) \Delta \tau\right)}{\varpi} \\
& +\int_{t_{1}}^{\sigma\left(t_{m}\right)} \Phi_{q}\left(\int_{s}^{t_{m}} h(\tau) \Phi_{p}\left(\frac{a \varpi}{h_{0}+\varpi M_{\eta}}\right) \Delta \tau\right) \Delta s \\
\leq & c
\end{aligned}
$$

which implies that $A(\overline{P(\gamma, c)}) \subset \overline{P(\gamma, c)}$. 
Advances in Difference Equations

11

Let $N=h_{0} / \varpi+M_{\eta}$ and

$$
\begin{aligned}
u_{1}= & \frac{b}{M_{Q}}\left(\frac{\Phi_{q}\left(\int_{t_{1}}^{t_{m}} h(\tau) \Delta \tau\right)-\sum_{i=1}^{m-2} \epsilon_{i} \int_{t_{1}}^{\xi_{i}} \Phi_{q}\left(\int_{s}^{t_{m}} h(\tau) \Delta \tau\right) \Delta s}{\varpi}\right) \\
& -\frac{b}{M_{Q}}\left(\frac{\sum_{j=1}^{n} \theta_{j} \Phi_{q}\left(\int_{\eta_{j}}^{t_{m}} h(\tau) \Delta \tau\right)}{\varpi}\right)+\frac{b}{M_{Q}} \int_{t_{1}}^{t} \Phi_{q}\left(\int_{s}^{t_{m}} h(\tau) \Delta \tau\right) \Delta s, \\
u_{2}= & \frac{a}{N}\left(\frac{\Phi_{q}\left(\int_{t_{1}}^{t_{m}} h(\tau) \Delta \tau\right)-\sum_{i=1}^{m-2} \epsilon_{i} \int_{t_{1}}^{\grave{s}_{i}} \Phi_{q}\left(\int_{s}^{t_{m}} h(\tau) \Delta \tau\right) \Delta s}{\varpi}\right) \\
& -\frac{a}{N}\left(\frac{\sum_{j=1}^{n} \theta_{j} \Phi_{q}\left(\int_{\eta_{j}}^{t_{m}} h(\tau) \Delta \tau\right)}{\varpi}\right)+\frac{a}{N} \int_{t_{1}}^{t} \Phi_{q}\left(\int_{s}^{t_{m}} h(\tau) \Delta \tau\right) \Delta s,
\end{aligned}
$$

we can verify that $u_{1}, u_{2} \in P$. By calculation,

$$
\begin{aligned}
\alpha\left(u_{1}\right)= & \frac{b}{M_{Q}}\left(\frac{\Phi_{q}\left(\int_{t_{1}}^{t_{m}} h(\tau) \Delta \tau\right)-\sum_{i=1}^{m-2} \epsilon_{i} \int_{t_{1}}^{\xi_{i}} \Phi_{q}\left(\int_{s}^{t_{m}} h(\tau) \Delta \tau\right) \Delta s}{\varpi}\right) \\
& -\frac{b}{M_{Q}}\left(\frac{\sum_{j=1}^{n} \theta_{j} \Phi_{q}\left(\int_{\eta_{j}}^{t_{m}} h(\tau) \Delta \tau\right)}{\varpi}\right)+\frac{b}{M_{Q}} \int_{t_{1}}^{\rho} \Phi_{q}\left(\int_{s}^{t_{m}} h(\tau) \Delta \tau\right) \Delta s \\
\geq & \frac{b}{M_{Q}}\left(\frac{1-\sum_{j=1}^{n} \theta_{j}-\sum_{i=1}^{m-2} \epsilon_{i} \xi_{i}}{\varpi} \Phi_{q}\left(\int_{t_{1}}^{t_{m}} h(\tau) \Delta \tau\right)+\int_{t_{1}}^{\rho} \Phi_{q}\left(\int_{s}^{t_{m}} h(\tau) \Delta \tau\right) \Delta s\right) \\
> & \frac{b}{M_{Q}} \int_{t_{1}}^{Q} \Phi_{q}\left(\int_{s}^{t_{m}} h(\tau) \Delta \tau\right) \Delta s=b, \\
\theta\left(u_{1}\right)= & \frac{b}{M_{Q}}\left(\frac{\Phi_{q}\left(\int_{t_{1}}^{t_{m}} h(\tau) \Delta \tau\right)-\sum_{i=1}^{m-2} \epsilon_{i} \int_{t_{1}}^{\xi_{i}} \Phi_{q}\left(\int_{s}^{t_{m}} h(\tau) \Delta \tau\right) \Delta s}{\varpi}\right) \\
& -\frac{b}{M_{Q}}\left(\frac{\sum_{j=1}^{n} \theta_{j} \Phi_{q}\left(\int_{\eta_{j}}^{t_{m}} h(\tau) \Delta \tau\right)}{\varpi}\right)+\frac{b}{M_{Q}} \int_{t_{1}}^{\eta} \Phi_{q}\left(\int_{s}^{t_{m}} h(\tau) \Delta \tau\right) \Delta s \\
\leq & \frac{b}{M_{Q}}\left(\frac{\Phi_{q}\left(\int_{t_{1}}^{t_{m}} h(\tau) \Delta \tau\right)}{\varpi}+\int_{t_{1}}^{\eta} \Phi_{q}\left(\int_{s}^{t_{m}} h(\tau) \Delta \tau\right) \Delta s\right) \leq k,
\end{aligned}
$$


12

Advances in Difference Equations

$$
\begin{aligned}
& \gamma\left(u_{1}\right)=\frac{b}{M_{Q}}\left(\frac{\Phi_{q}\left(\int_{t_{1}}^{t_{m}} h(\tau) \Delta \tau\right)-\sum_{i=1}^{m-2} \epsilon_{i} \int_{t_{1}}^{\xi_{i}} \Phi_{q}\left(\int_{s}^{t_{m}} h(\tau) \Delta \tau\right) \Delta s}{\varpi}\right) \\
& -\frac{b}{M_{Q}}\left(\frac{\sum_{j=1}^{n} \theta_{j} \Phi_{q}\left(\int_{\eta_{j}}^{t_{m}} h(\tau) \Delta \tau\right)}{\varpi}\right)+\frac{b}{M_{Q}} \int_{t_{1}}^{\sigma\left(t_{m}\right)} \Phi_{q}\left(\int_{s}^{t_{m}} h(\tau) \Delta \tau\right) \Delta s \\
& \leq \frac{b}{M_{Q}}\left(\frac{\Phi_{q}\left(\int_{t_{1}}^{t_{m}} h(\tau) \Delta \tau\right)}{\varpi}+\int_{t_{1}}^{\sigma\left(t_{m}\right)} \Phi_{q}\left(\int_{s}^{t_{m}} h(\tau) \Delta \tau\right) \Delta s\right) \leq c, \\
& \beta\left(u_{2}\right)=\frac{a}{N}\left(\frac{\Phi_{q}\left(\int_{t_{1}}^{t_{m}} h(\tau) \Delta \tau\right)-\sum_{i=1}^{m-2} \epsilon_{i} \int_{t_{1}}^{\xi_{i}} \Phi_{q}\left(\int_{s}^{t_{m}} h(\tau) \Delta \tau\right) \Delta s}{\varpi}\right) \\
& -\frac{a}{N}\left(\frac{\sum_{j=1}^{n} \theta_{j} \Phi_{q}\left(\int_{\eta_{j}}^{t_{m}} h(\tau) \Delta \tau\right)}{\varpi}\right)+\frac{a}{N} \int_{t_{1}}^{\eta} \Phi_{q}\left(\int_{s}^{t_{m}} h(\tau) \Delta \tau\right) \Delta s \\
& <\frac{a}{N}\left(\frac{\Phi_{q}\left(\int_{t_{1}}^{t_{m}} h(\tau) \Delta \tau\right)}{\varpi}+\int_{t_{1}}^{\eta} \Phi_{q}\left(\int_{s}^{t_{m}} h(\tau) \Delta \tau\right) \Delta s\right)=a, \\
& \varphi\left(u_{2}\right)=\frac{a}{N}\left(\frac{\Phi_{q}\left(\int_{t_{1}}^{t_{m}} h(\tau) \Delta \tau\right)-\sum_{i=1}^{m-2} \epsilon_{i} \int_{t_{1}}^{\xi_{i}} \Phi_{q}\left(\int_{s}^{t_{m}} h(\tau) \Delta \tau\right) \Delta s}{\varpi}\right) \\
& -\frac{a}{N}\left(\frac{\sum_{j=1}^{n} \theta_{j} \Phi_{q}\left(\int_{\eta_{j}}^{t_{m}} h(\tau) \Delta \tau\right)}{\varpi}\right)+\frac{a}{N} \int_{t_{1}}^{\rho} \Phi_{q}\left(\int_{s}^{t_{m}} h(\tau) \Delta \tau\right) \Delta s \\
& \geq \frac{a}{N}\left(\frac{1-\sum_{j=1}^{n} \theta_{j}-\sum_{i=1}^{m-2} \epsilon_{i} \xi_{i}}{\varpi} \Phi_{q}\left(\int_{t_{1}}^{t_{m}} h(\tau) \Delta \tau\right)+\int_{t_{1}}^{\rho} \Phi_{q}\left(\int_{s}^{t_{m}} h(\tau) \Delta \tau\right) \Delta S\right) \\
& >\frac{a}{N} \int_{t_{1}}^{Q} \Phi_{q}\left(\int_{s}^{t_{m}} h(\tau) \Delta \tau\right) \Delta s>h, \\
& \gamma\left(u_{2}\right)=\frac{a}{N}\left(\frac{\Phi_{q}\left(\int_{t_{1}}^{t_{m}} h(\tau) \Delta \tau\right)-\sum_{i=1}^{m-2} \epsilon_{i} \int_{t_{1}}^{\xi_{i}} \Phi_{q}\left(\int_{s}^{t_{m}} h(\tau) \Delta \tau\right) \Delta s}{\varpi}\right) \\
& -\frac{a}{N}\left(\frac{\sum_{j=1}^{n} \theta_{j} \Phi_{q}\left(\int_{\eta_{j}}^{t_{m}} h(\tau) \Delta \tau\right)}{\varpi}\right)+\frac{a}{N} \int_{t_{1}}^{\sigma\left(t_{m}\right)} \Phi_{q}\left(\int_{s}^{t_{m}} h(\tau) \Delta \tau\right) \Delta s \\
& \leq \frac{a}{N}\left[\frac{\Phi_{q}\left(\int_{t_{1}}^{t_{m}} h(\tau) \Delta \tau\right)}{\varpi}+\int_{t_{1}}^{\sigma\left(t_{m}\right)} \Phi_{q}\left(\int_{s}^{t_{m}} h(\tau) \Delta \tau\right) \Delta s\right]<c .
\end{aligned}
$$


So, $u_{1} \in P(\gamma, \theta, \alpha, b, k, c), \alpha\left(u_{1}\right)>b, u_{2} \in Q(\gamma, \beta, \varphi, h, a, c), \beta\left(u_{2}\right)<a$, which means that $\{u \in P(\gamma, \theta, \alpha, b, k, c): \alpha(u)>b\}$ and $\{u \in Q(\gamma, \beta, \varphi, h, a, c): \beta(u)<a\}$ are not empty.

For $u \in P(\gamma, \theta, \alpha, b, k, c)$,

$$
\begin{aligned}
\alpha(A u)= & \frac{\Phi_{q}\left(\int_{t_{1}}^{t_{m}} h(\tau) f(\tau, u(\tau)) \Delta \tau\right)-\sum_{i=1}^{m-2} \epsilon_{i} \int_{t_{1}}^{\xi_{i}} \Phi_{q}\left(\int_{s}^{t_{m}} h(\tau) f(\tau, u(\tau)) \Delta \tau\right) \Delta s}{\varpi} \\
& -\frac{\sum_{j=1}^{n} \theta_{j} \Phi_{q}\left(\int_{\eta_{j}}^{t_{m}} h(\tau) f(\tau, u(\tau)) \Delta \tau\right)}{\varpi}+\int_{t_{1}}^{\rho} \Phi_{q}\left(\int_{s}^{t_{m}} h(\tau) f(\tau, u(\tau)) \Delta \tau\right) \Delta s \\
\geq & \frac{1-\sum_{j=1}^{n} \theta_{j}-\sum_{i=1}^{m-2} \epsilon_{i} \xi_{i}}{\varpi} \Phi_{q}\left(\int_{t_{1}}^{t_{m}} h(\tau) f(\tau, u(\tau)) \Delta \tau\right) \\
& +\int_{t_{1}}^{Q} \Phi_{q}\left(\int_{s}^{t_{m}} h(\tau) f(\tau, u(\tau)) \Delta \tau\right) \Delta s \\
> & \int_{t_{1}}^{\rho} \Phi_{q}\left(\int_{Q}^{\eta} h(\tau) f(\tau, u(\tau)) \Delta \tau\right) \Delta s \\
\geq & \int_{t_{1}}^{\rho} \Phi_{q}\left(\int_{\rho}^{\eta} h(\tau) \Phi_{p}\left(\frac{b}{M_{0}}\right) \Delta \tau\right) \Delta s=b
\end{aligned}
$$

and for $u \in Q(\gamma, \beta, \varphi, h, a, c)$,

$$
\begin{aligned}
\beta(A u)= & \frac{\Phi_{q}\left(\int_{t_{1}}^{t_{m}} h(\tau) f(\tau, u(\tau)) \Delta \tau\right)-\sum_{i=1}^{m-2} \epsilon_{i} \int_{t_{1}}^{\xi_{i}} \Phi_{q}\left(\int_{s}^{t_{m}} h(\tau) f(\tau, u(\tau)) \Delta \tau\right) \Delta s}{\varpi} \\
& -\frac{\sum_{j=1}^{n} \theta_{j} \Phi_{q}\left(\int_{\eta_{j}}^{t_{m}} h(\tau) f(\tau, u(\tau)) \Delta \tau\right)}{\varpi}+\int_{t_{1}}^{\eta} \Phi_{q}\left(\int_{s}^{t_{m}} h(\tau) f(\tau, u(\tau)) \Delta \tau\right) \Delta s \\
\leq & \frac{\Phi_{q}\left(\int_{t_{1}}^{t_{m}} h(\tau) f(\tau, u(\tau)) \Delta \tau\right)}{\varpi}+\int_{t_{1}}^{\eta} \Phi_{q}\left(\int_{s}^{t_{m}} h(\tau) f(\tau, u(\tau)) \Delta \tau\right) \Delta s \\
< & \frac{\Phi_{q}\left(\int_{t_{1}}^{t_{m}} h(\tau) \Phi_{p}\left(a \varpi /\left(h_{0}+\varpi M_{\eta}\right)\right) \Delta \tau\right)}{\varpi}+\int_{t_{1}}^{\eta} \Phi_{q}\left(\int_{s}^{t_{m}} h(\tau) \Phi_{p}\left(\frac{a \varpi}{h_{0}+\varpi M_{\eta}}\right) \Delta \tau\right) \triangle s=a .
\end{aligned}
$$

Thus (i) and (ii) in Lemma 2.4 hold.

On the other hand, for $u \in P(\gamma, \alpha, b, c)$ with $\theta(A u)>k$, we have $\alpha(A u)=A u(\rho) \geq$ $\left(\left(\rho-t_{1}\right) /\left(\eta-t_{1}\right)\right)(A u(\eta))=\left(\left(\rho-t_{1}\right) /\left(\eta-t_{1}\right)\right) \theta(A u)>\left(\left(\rho-t_{1}\right) /\left(\eta-t_{1}\right)\right) k>b$. And for $u \in P(\gamma, \beta, a, c)$ with $\varphi(A u)<h$, we can obtain $\beta(A u)=A u(\eta) \leq\left(\left(\eta-t_{1}\right) /\left(\rho-t_{1}\right)\right) A u(\rho)=$ $\left(\left(\eta-t_{1}\right) /\left(\rho-t_{1}\right)\right) \varphi(A u)<\left(\left(\eta-t_{1}\right) /\left(\rho-t_{1}\right)\right) h<a$. Thus, (iii) and (iv) in Lemma 2.4 hold. 
So, by Lemma 2.4, we obtain that the BVP (1.3) has at least three positive solutions $u_{1}, u_{2}, u_{3} \in \overline{P(\gamma, c)}$ such that

$$
\max _{t \in[Q, \eta]_{\mathbb{T}}} u_{1}(t)<a<\max _{t \in[Q, \eta]_{\mathbb{T}}} u_{3}(t), \quad \min _{t \in[\rho, \eta]_{\mathbb{T}}} u_{3}(t)<b<\min _{t \in[\rho, \eta]_{\mathbb{T}}} u_{2}(t)
$$

This completes the proof.

Remark 3.4. Let $R=c, r=b, v=k$, we can find that the conditions of Theorem 3.1 are contained in Theorem 3.3.

Example 3.5. Let $\mathbb{T}=\{0.1,0.18\} \cup[0.2,1] \cup\{1.2\} \cup[1.5,2], p=2$, consider the following eightpoint BVP:

$$
\begin{gathered}
\left(\Phi_{p}\left(u^{\Delta}\right)\right)^{\Delta}(t)+h(t) f(t, u(t))=0, \quad t \in(0.1,2)_{\mathbb{T}} \\
u^{\Delta}(0.1)-\sum_{j=1}^{3} \theta_{j} u^{\Delta}\left(\eta_{j}\right)-\sum_{i=1}^{3} \epsilon_{i} u\left(\xi_{i}\right)=0, \quad u^{\Delta}(2)=0,
\end{gathered}
$$

where $h(t)=t+\sigma(t), \theta_{1}=1 / 12, \theta_{2}=1 / 7, \theta_{3}=1 / 42, \epsilon_{1}=1 / 6, \epsilon_{2}=1 / 24, \epsilon_{3}=1 / 8, \xi_{1}=0.33$, $\xi_{2}=0.45, \xi_{3}=1.65, \eta_{1}=0.88, \eta_{2}=1.86, \eta_{3}=1.95$, for all $t \in \mathbb{T}$, and

$$
f(t, u)= \begin{cases}0.00015, & {[0.4,1.8]_{\mathbb{T}} \times[0.0001,0.055]} \\ g(u), & \text { other }\end{cases}
$$

where $g(u)$ is continuous, $0 \leq g(u) \leq 0.0026$, and $g(0.0001)=g(0.055)=0.00015$.

Set $\varrho=0.4, \eta=1.8$, by calculation,

$$
\begin{gathered}
\sum_{j=1}^{3} \theta_{j}=\frac{1}{4}, \quad \sum_{i=1}^{3} \epsilon_{i}=\frac{1}{3}, \quad h_{0}=3.99, \quad M_{0}=0.924, \\
M_{Q}=\frac{3.539656}{3}, \quad M_{\eta}=\frac{15.050656}{3}, \quad M_{\sigma\left(t_{m}\right)}=\frac{15.282656}{3},
\end{gathered}
$$

and let $b=0.0001, k=0.055, c=0.102585312, a=0.045, h=0.000125$. Clearly, we can verify that the conditions in Theorem 3.3 are fulfilled. Thus, by Theorem 3.3, the BVP (3.21) has at least three positive solutions $u_{1}, u_{2}$ and $u_{3}$ such that

$$
\max _{t \in[\rho, \eta]_{\mathbb{T}}} u_{1}(t)<0.045<\max _{t \in[\rho, \eta]_{\mathbb{T}}} u_{3}(t), \quad \min _{t \in[\rho, \eta]_{\mathbb{T}}} u_{3}(t)<0.0001<\min _{t \in[\rho, \eta]_{\mathbb{T}}} u_{2}(t) .
$$

Remark 3.6. If we let $R=0.102585312, r=0.0001, \iota=r+10^{-5}, v=0.055$, we can also verify that the conditions in Theorem 3.1 are satisfied. 


\section{Acknowledgments}

This work is supported by the Natural Science Foundation of Ludong University (24200301, 24070301, 24070302), Program for Innovative Research Team in Ludong University, and a Project of Shandong Province Higher Educational Science and Technology Program.

\section{References}

[1] D. Anderson, R. Avery, and J. Henderson, "Existence of solutions for a one dimensional $p$-Laplacian on time-scales," Journal of Difference Equations and Applications, vol. 10, no. 10, pp. 889-896, 2004.

[2] M. Feng, H. Feng, X. Zhang, and W. Ge, "Triple positive solutions for a class of $m$-point dynamic equations on time scales with $p$-Laplacian," Mathematical and Computer Modelling, vol. 48, no. 7-8, pp. 1213-1226, 2008.

[3] Y. Sang and H. Su, "Several existence theorems of nonlinear $m$-point boundary value problem for p-Laplacian dynamic equations on time scales," Journal of Mathematical Analysis and Applications, vol. 340, no. 2, pp. 1012-1026, 2008.

[4] C. Song and P. Weng, "Multiple positive solutions for $p$-Laplacian functional dynamic equations on time scales," Nonlinear Analysis: Theory, Methods \& Applications, vol. 68, no. 1, pp. 208-215, 2008.

[5] F. Cao, Z. Han, and S. Sun, "Existence of periodic solutions for $p$-Laplacian equations on time scales," Advances in Difference Equations, vol. 2010, no. 1, Article ID 584375, 13 pages, 2010.

[6] H.-R. Sun and W.-T. Li, "Multiple positive solutions for $p$-Laplacian $m$-point boundary value problems on time scales," Applied Mathematics and Computation, vol. 182, no. 1, pp. 478-491, 2006.

[7] H.-R. Sun and W.-T. Li, "Existence theory for positive solutions to one-dimensional $p$-Laplacian boundary value problems on time scales," Journal of Differential Equations, vol. 240, no. 2, pp. $217-$ $248,2007$.

[8] J. Yang and Z. Wei, "Existence of positive solutions for fourth-order $m$-point boundary value problems with a one-dimensional $p$-Laplacian operator," Nonlinear Analysis: Theory, Methods E Applications, vol. 71, no. 7-8, pp. 2985-2996, 2009.

[9] J. Zhao and W. Ge, "Existence results of $2 m$-point boundary value problem of Sturm-Liouville type with sign changing nonlinearity," Mathematical and Computer Modelling, vol. 49, no. 5-6, pp. 946-954, 2009.

[10] Y. Zhu and J. Zhu, "The multiple positive solutions for $p$-Laplacian multipoint BVP with sign changing nonlinearity on time scales," Journal of Mathematical Analysis and Applications, vol. 344, no. 2, pp. 616-626, 2008.

[11] Z. He, "Existence of two solutions of $m$-point boundary value problem for second order dynamic equations on time scales," Journal of Mathematical Analysis and Applications, vol. 296, no. 1, pp. 97-109, 2004.

[12] M. Bohner and A. Peterson, Dynamic Equations on Time Scales: An Introduction with Applications, Birkhäuser, Boston, Mass, USA, 2001.

[13] V. Lakshmikantham, S. Sivasundaram, and B. Kaymakcalan, Dynamic Systems on Measure Chains, vol. 370 of Mathematics and Its Applications, Kluwer Academic Publishers, Dordrecht, The Netherlands, 1996.

[14] R. P. Agarwal and D. O'Regan, "A generalization of the Petryshyn-Leggett-Williams fixed point theorem with applications to integral inclusions," Applied Mathematics and Computation, vol. 123, no. 2, pp. 263-274, 2001.

[15] R. P. Agarwal and M. Bohner, "Basic calculus on time scales and some of its applications," Results in Mathematics, vol. 35, no. 1-2, pp. 3-22, 1999.

[16] R. Avery, J. Henderson, and D. O'Regan, "Four functionals fixed point theorem," Mathematical and Computer Modelling, vol. 48, no. 7-8, pp. 1081-1089, 2008.

[17] R. I. Avery, "A generalization of the Leggett-Williams fixed point theorem," Mathematical Sciences Research Hot-Line, vol. 3, no. 7, pp. 9-14, 1999.

[18] R. P. Agarwal, M. Bohner, and P. Řehák, "Half-linear dynamic equations," in Nonlinear Analysis and Applications: to V. Lakshmikantham on His 80th Birthday. Vol. 1, pp. 1-57, Kluwer Academic Publishers, Dordrecht, The Netherlands, 2003.

[19] B. Aulbach and L. Neidhart, "Integration on measure chains," in Proceedings of the 6th International Conference on Difference Equations, pp. 239-252, CRC, Boca Raton, Fla, USA. 